\title{
Detecting Cortical Spreading Depolarization with Full Band Scalp Electroencephalography: An Illusion?
}

\author{
Jeannette Hofmeijer ${ }^{1,2 *}$, C. R. van Kaam ${ }^{1,2}$, Babette van de Werff ${ }^{1,2}$, Sarah E. Vermeer ${ }^{2}$, \\ Marleen C. Tjepkema-Cloostermans ${ }^{3}$ and Michel J. A. M. van Putten ${ }^{1,3}$

\begin{abstract}
'Department of Clinical Neurophysiology, MIRA Institute for Biomedical Technology and Technical Medicine, University of Twente, Enschede, Netherlands, ${ }^{2}$ Department of Neurology, Rijnstate Hospital, Arnhem, Netherlands, ${ }^{3}$ Department of Clinical Neurophysiology, Medisch Spectrum Twente, Enschede, Netherlands
\end{abstract}

\section{OPEN ACCESS}

Edited by:

Nikolaus Plesnila,

Institute for Stroke and

Dementia Research, Germany

Reviewed by:

Jens P. Dreier,

Charité Universitätsmedizin

Berlin, Germany

Vincent Thijs,

Florey Institute of Neuroscience and Mental Health, Australia

*Correspondence: Jeannette Hofmeijer jhofmeijer@rijnstate.n

Specialty section: This article was submitted to Stroke, a section of the journal Frontiers in Neurology

Received: 27 October 2017 Accepted: 10 January 2018 Published: 25 January 2018

Citation:

Hofmeijer J, van Kaam CR, van de Werff B, Vermeer SE, Tjepkema-Cloostermans MC and van Putten MJAM (2018) Detecting

Cortical Spreading Depolarization with Full Band Scalp

Electroencephalography: An Illusion? Front. Neurol. 9:17.

doi: 10.3389/fneur.2018.00017
Introduction: There is strong evidence suggesting detrimental effects of cortical spreading depolarization (CSD) in patients with acute ischemic stroke and severe traumatic brain injury. Previous studies implicated scalp electroencephalography (EEG) features to be correlates of CSD based on retrospective analysis of EEG epochs after having detected "CSD" in time aligned electrocorticography. We studied the feasibility of CSD detection in a prospective cohort study with continuous EEG in 18 patients with acute ischemic stroke and 18 with acute severe traumatic brain injury.

Methods: Full band EEG with 21 silver/silver chloride electrodes was started within $48 \mathrm{~h}$ since symptom onset. Five additional electrodes were used above the infarct. We visually analyzed all raw EEG data in epochs of $1 \mathrm{~h}$. Inspection was directed at detection of the typical combination of CSD characteristics, i.e., (i) a large slow potential change (SPC) accompanied by a simultaneous amplitude depression of $>1 \mathrm{~Hz}$ activity, (ii) focal presentation, and (iii) spread reflected as appearance on neighboring electrodes with a delay.

Results: In 3,035 one-hour EEG epochs, infraslow activity (ISA) was present in half to three quarters of the registration time. Typically, activity was intermittent with amplitudes of 40-220 $\mu \mathrm{V}$, approximately half was oscillatory. There was no specific spatial distribution. Relevant changes of ISA were always visible in multiple electrodes, and not focal, as expected in CSD. ISA appearing as "SPC" was mostly associated with an amplitude increase of faster activities, and never with suppression. In all patients, depressions of spontaneous brain activity occurred. However, these were not accompanied by simultaneous SPC, occurred simultaneously on all channels, and were not focal, let alone spread, as expected in CSD.

Conclusion: With full band scalp EEG in patients with cortical ischemic stroke or traumatic brain injury, we observed various ISA, probably modulating cortical excitability. However, we were unable to identify unambiguous characteristics of CSD.

Keywords: cortical spreading depolarization, infraslow activity, acute ischemic stroke, traumatic brain injury, full band electroencephalography

Abbreviations: COSBID, Co-Operative Study on Brain Injury Depolarizations; CSD, cortical spreading depolarization; ECoG, electrocorticography; ISA, infraslow activity $(0.001-0.1 \mathrm{~Hz})$; mRS, modified Rankin Scale; NIHSS, National Institutes of Health Stroke Scale. 


\section{INTRODUCTION}

Cortical spreading depolarization (CSD) is a wave of neuronal and glial depolarization, propagating across the brain's gray matter at a velocity of $2-5 \mathrm{~mm} / \mathrm{min}$ (1). CSD waves are associated with failure of brain ion homeostasis and lead to silencing of spontaneous and evoked synaptic transmission during $3 \mathrm{~min}$ under relatively healthy conditions to many hours in metabolically comprised tissue (2). The subsequent correction of ion gradients is accompanied with increased energy use $(3,4)$. CSD is a benign phenomenon in "healthy" brains with preserved perfusion and metabolism (5). As such, it plays a role in migraine aura, where the initial depolarization wave induces a wave of relative hyperperfusion called "spreading hyperemia" (6-9). Otherwise, in acutely injured brain tissue, with impaired cerebrovascular autoregulation and exhausted energy metabolism, CSD can induce spreading ischemia, which delays the neuronal repolarization and hence the recovery from the CSD-induced toxic intraneuronal changes, thereby increasing the risk of irreversible damage (10-13).

There is strong experimental and clinical evidence suggesting presence and potential detrimental effects of CSD in most, if not all, patients with acute cortical ischemic stroke, severe traumatic brain injury, and subarachnoid hemorrhage $(10,11,14-21)$. With prevention of CSD being a possible treatment target, real time clinical measurement would be useful to guide eventual treatments. The Co-Operative Study on Brain Injury Depolarizations (COSBID) study group succeeded to detect CSD signatures with invasive subdural electrocorticography (ECoG) in patients with "malignant" ischemic stroke (14), traumatic brain injury (15), subarachnoid hemorrhage (22), and spontaneous intracerebral hemorrhage $(10,23)$. In these studies, CSD was defined as the combination of slow potential change (SPC) with simultaneous transient suppression of faster activities, propagating along the cortex. In the absence of any ECoG background activity (continuous depressions), which is a common situation in, for example, the ischemic penumbra, SPCs alone were designated as "silent" or "isoelectric" "peri-infarct depolarizations" (10).

For wide spread clinical application, reliable CSD detection should optimally be possible in a non-invasive way. The COSBID group implicated specific scalp electroencephalography (EEG) features to be correlates of CSD based on EEG studies combined with ECoG (24). Hereby, scalp EEG signatures were retrospectively sought for after having detected CSD in ECoG data. On EEG, SPC with a median amplitude of around $-270 \mu \mathrm{V}$ and duration of approximately $5.5 \mathrm{~min}$, accompanied by suppression of faster activities, were adjudicated as CSD (24). In contrast to ECoG, only clusters of (and not isolated) CSD were identified, and EEG allowed no visualization of the typical spread. Still, it was concluded that (spreading) depolarizations and depressions of spontaneous activity in time-compressed human scalp EEG could serve as non-invasive signatures of spreading depolarizations and depressions.

We aim to study the feasibility of CSD detection, including its clinical relevance, by continuous full band scalp EEG in prospective cohort studies of patients with acute ischemic stroke and traumatic brain injury.

\section{MATERIALS AND METHODS}

\section{Design}

We performed prospective cohort studies with continuous EEG monitoring for detection of CSD in patients with acute cortical ischemic stroke and acute traumatic brain injury. Patients with ischemic stroke were included at stroke units of two teaching hospitals. In Rijnstate Hospital, Arnhem, patients were included between February 2016 and October 2016; in Medisch Spectrum Twente, Enschede, between November 2014 and May 2015. Patients with acute traumatic brain injury were included at the intensive care unit of Medisch Spectrum Twente between November 2015 and December 2016.

\section{Approval and Consent}

The Medical Research Ethics Committee Twente approved the research protocol for monitoring and follow-up of patients with ischemic stroke in October 2014 (registry number NL50284.044.14). Written and informed consent for continuous EEG monitoring and follow-up of patients with ischemic stroke was obtained from the patient or a legal representative, in case of a decreased consciousness or severe aphasia. Since continuous EEG monitoring and clinical follow-up of patients with a decreased consciousness are part of current care on the intensive care unit of MST, the Medical Research Ethics Committee Twente waived the need for informed consent in patients with acute traumatic brain injury.

\section{Patients}

Inclusion criteria for patients with acute ischemic stroke were age $\geq 18$ years, clinical symptoms consistent with a cortical localization, and severity leading to a score of $\geq 4$ on the National Institutes of Health Stroke Scale (NIHSS). Exclusion criteria consisted of any progressive brain illness, expectation of short-term death due to stroke, and a pre-stroke modified Rankin Scale (mRS) score of $>2$. Treatment was according to standard protocols for patients with a brain infarct at the stroke unit, including early mobilization, if possible. Interference between the EEG recording and early mobilization was minimized by the use of small, mobile EEG equipment, if necessary. Inclusion criteria for patients with traumatic brain injury were admission to the intensive care unit with moderate to severe traumatic brain injury [Glasgow Coma Scale (GCS) $\leq 12$ ] and age $\geq 18$ years. Exclusion criteria were concomitant cardiac arrest or large open wounds, which hamper EEG registration. Treatment was according to standard protocols.

\section{Outcome}

To relate eventual CSD candidates to clinical outcome, outcome measures were predefined and collected prospectively. The primary outcome measure for patients with ischemic stroke was functional outcome as expressed by the score on the mRS at 3 months after stroke. Secondary outcome measures included secondary deterioration during hospital admission, defined as an increase on the NIHSS score of two points or more. The NIHSS score was collected at the first presentation at the emergency room, at the start of EEG registration, every 
morning, at the end of EEG registration, and with any sign of neurological deterioration. The primary outcome measure for patients with traumatic brain injury was functional outcome as expressed by Extended Glasgow Outcome Scale at hospital discharge.

\section{EEG Registration}

Electroencephalography registration started as soon as possible after admission to the stroke or intensive care unit, and within $48 \mathrm{~h}$ after symptom onset. For stroke patients, registration was aborted after 3 days, at discharge, or when the patient requested so, for example, in case of discomfort. In traumatic brain injury patients, registration was aborted after 7 days, at discharge, or when the patient regained consciousness. A Neurocenter EEG system (Clinical Science Systems, Leiden, the Netherlands) or (for mobile stroke patients) a portable Mobita EEG system (TMS International, Oldenzaal, the Netherlands) was used, with a DC-coupled amplifier (TMS International, Oldenzaal, the Netherlands, with the same settings for stationary and portable systems) and a sample frequency of 256 or $250 \mathrm{~Hz}$. Twenty-one silver/silver chloride electrodes were placed on the scalp according to the international 10-20 system. In patients with ischemic stroke, five additional electrodes were placed above the infarcted area. These were placed on positions FC4, FC6, C6, CP6, and CP4 for right-sided, and FC3, FC5, C5, CP5, and CP3 for left sided infarcts. Electrodes were attached using collodion glue and filled with Ten 20 conductive paste. Electrode impedance was controlled twice a day and kept below $5 \mathrm{k} \Omega$. Recordings were obtained with the common reference.

\section{EEG Analysis}

Signal processing was done with Matlab (MATLAB R2016a, The MathWorks Inc., Natick, MA, USA). Full band EEG recordings were stored in EDF+ format using the method described in Kemp et al. (25). Before further analysis, all EEGs were visually inspected in the frequency band of $1-35 \mathrm{~Hz}$ to assess the general quality of the recording. Epochs with loose electrodes and major artifacts were discarded.

Electroencephalography analysis was qualitative, by visual inspection. The search for CSD was done by inspection of all raw EEG data in epochs of $1 \mathrm{~h}$. Data from patients with brain infarcts were inspected with bipolar electrode pairs, including four pairs including the additional electrodes above the infarcted hemisphere. Data from patients with traumatic brain injury, the Laplacian source derivation was used. For each electrode pair or electrode, the infraslow activity (ISA) $(0.001-0.1 \mathrm{~Hz}$ ), the "conventional" EEG activity $(0.5-30 \mathrm{~Hz})$, and the power of conventional activity were plotted. Stroke data were additionally inspected in the delta $(1-4 \mathrm{~Hz})$, theta $(5-7 \mathrm{~Hz})$, alpha $(8-12 \mathrm{~Hz})$, and beta $(13-30 \mathrm{~Hz})$ frequency bands. Filtering was achieved with a zero-phase second-order Butterworth bandpass filter (using the Matlab command "filtfilt"). Power was calculated every $30 \mathrm{~s}$ using a sliding window with the size of $60 \mathrm{~s}$. The power spectral density was estimated using Welch's method with a frequency resolution of $0.1 \mathrm{~Hz}$, Hamming window, and $50 \%$ overlap (26). Because the ISA filter had a start-up effect, an extra 10 min preceding the $1 \mathrm{~h}$ section was always added and removed before visualization.
Visual inspection was directed at a qualitative description of ISA and detection of the typical combination of CSD characteristics. These consist of (i) a large SPC $(=0.1 \mathrm{~Hz})$ accompanied by a simultaneous amplitude depression of spontaneous $>1 \mathrm{~Hz}$ activity, (ii) focal presentation of SPC and depression, and (iii) a spread of SPC and depression reflected as appearance on neighboring electrodes with a temporal delay. Sizes of the phenomena were based on previous studies, where the targeted SPC in the EEG had a median amplitude of 270-305 $\mu \mathrm{V}$ (range: 107-517) and a median duration of 5.5-7.4 min (IQR: 4.4-8.3) (24). As for depression of spontaneous brain activity, a median of 57\% (IQR 44-67) and a duration of 21 min (median; IQR 16-33) have been reported (27).

\section{RESULTS}

\section{Patients}

We included 18 patients with acute ischemic stroke, 11 in Rijnstate hospital, and 7 in MST. Baseline characteristics and outcomes are summarized in Table 1. The mean age was 72 (SD 11), and 44\% was female. The median NIHSS on admission was 12 (IQR 7). All patients had an infarct in the middle cerebral artery (MCA) territory. EEG registration started after a median of $9 \mathrm{~h}$ after symptom onset (IQR 10). In five patients, worsening of neurological deficit was observed during EEG administration, four remained stable, and nine improved. In 13 stroke patients, registrations were discontinued because of discomfort. After 3 months, 14 patients had a poor outcome, of whom three had died. We included 18 patients with acute traumatic brain injury. Baseline characteristics and outcomes are summarized in Table 2. The mean age was 51 (SD 19 ), and $22 \%$ was female. The median GCS score on the site of trauma 7. EEG registration started after a median of $17 \mathrm{~h}$ after trauma. Six patients died on the intensive care unit.

\section{Qualitative Description of ISA in Relation to "Conventional" EEG Activity}

We inspected 429 one-hour EEG epochs and an additional 429 overviews of extra electrodes above the infarcted area of the patients with infarcts. Approximately $40 \%$ of the epochs appeared to contain too may artifacts in the ISA band to further analyze. From patients with traumatic brain injury, 2,177 one-hour EEG epochs were inspected. A quarter contained too may artifacts in the ISA band to further analyze. In the remaining samples of both patient groups, ISA was present in half to three quarters of the registration time. Typically, activity was intermittent, where several minutes with abundant ISA were interspersed with minutes without ISA. Amplitudes were between 40 and $220 \mu \mathrm{V}$ and frequencies from the complete ISA spectrum were observed. Although the predominance of ISA varied between electrodes, there was no specific spatial distribution. ISA was often associated with simultaneous amplitude fluctuations of faster activities (Figures 1-3).

In five patients with brain infarcts and all patients with traumatic brain injury, periods of high amplitude $(>100 \mu \mathrm{V})$ oscillatory ISA was observed. The duration of this oscillatory activity varied from 30 min to many hours. Frequencies typically 
TABLE 1 | Baseline characteristics and outcome of patients with acute cortical ischemic stroke.

\begin{tabular}{|c|c|c|c|c|c|c|c|}
\hline Patient & $\begin{array}{l}\text { Age range } \\
\text { (years) }\end{array}$ & Stroke location & $\begin{array}{c}\text { EEG start } \\
\text { (h after stroke) }\end{array}$ & $\begin{array}{c}\text { Total EEG monitoring } \\
\text { time (h) }\end{array}$ & $\begin{array}{l}\text { NIHSS score } \\
\text { admission }\end{array}$ & $\begin{array}{l}\text { Deterioration time } \\
\text { (h after stroke) }\end{array}$ & mRS 3 months \\
\hline 1 & $75-89$ & MCA R & 1 & 22 & 11 & & 4 \\
\hline 2 & $70-74$ & MCA R & 18 & 45 & 17 & & 5 \\
\hline 3 & 85-89 & MCA L & 17 & 42 & 18 & 22 & 5 \\
\hline 4 & $50-54$ & MCA R & 9 & 18 & 10 & 13 & 2 \\
\hline 5 & $70-74$ & MCA R & 6 & 63 & 16 & & 3 \\
\hline 6 & $70-74$ & MCA L & 8 & 21 & 15 & 31 & 3 \\
\hline 7 & $65-69$ & MCA L & 5 & 17 & 8 & & 2 \\
\hline 8 & $65-69$ & MCA R & 18 & 16 & 4 & 11 & 5 \\
\hline 9 & $45-49$ & MCA L & 15 & 2 & 21 & & 2 \\
\hline 10 & $70-74$ & MCA R & 13 & 22 & 11 & & 5 \\
\hline 11 & $75-79$ & MCA L & 18 & 20 & 12 & & 4 \\
\hline 12 & 80-84 & MCA L & 8 & 11 & 10 & & 4 \\
\hline 13 & $75-79$ & MCA R & 6 & 20 & 15 & 6 & 6 \\
\hline 14 & $85-89$ & MCA R & 10 & 21 & 12 & & 1 \\
\hline 15 & $70-74$ & MCA L & 7 & 26 & 20 & 26 & 6 \\
\hline 16 & 85-89 & MCA R & 35 & 22 & 9 & & 6 \\
\hline 17 & $65-69$ & MCA L & 7 & 44 & 10 & 23 & 5 \\
\hline 18 & $50-54$ & MCA L & 6 & 15 & 23 & & 3 \\
\hline
\end{tabular}

M, male; F, female; MCA, middle cerebral artery; R, right; L, left; EEG, electroencephalography; NIHSS, National Institutes of Health Stroke Scale; mRS, modified Rankin Scale; mRS of 6 , death.

TABLE 2 | Baseline characteristics and outcome of patients with acute traumatic brain injury.

\begin{tabular}{|c|c|c|c|c|c|c|}
\hline Patient & Age range (years) & CT abnormalities & $\begin{array}{c}\text { EEG start } \\
\text { (h after trauma) }\end{array}$ & Total EEG monitoring time (h) & $\begin{array}{c}\text { GCS at trauma } \\
\text { site }\end{array}$ & $\begin{array}{l}\text { GOSE at } \\
\text { discharge }\end{array}$ \\
\hline 1 & $25-29$ & Bilateral & 7 & 214 & 8 & 3 \\
\hline 2 & $22-24$ & Bilateral and infratentorial & 25 & 170 & 4 & 3 \\
\hline 3 & $40-44$ & Left hemishere & 17 & 139 & 7 & 2 \\
\hline 4 & $70-74$ & Left hemishere & 8 & 209 & 10 & 1 \\
\hline 5 & $55-59$ & Bilateral & 7 & 137 & 7 & 3 \\
\hline 6 & $55-59$ & Bilateral and infratentorial & 16 & 172 & 3 & 1 \\
\hline 7 & $15-19$ & Left hemisphere and infratentorial & 17 & 168 & 8 & 4 \\
\hline 8 & $60-64$ & Bilateral & 7 & 173 & NA & 4 \\
\hline 9 & $20-24$ & Right hemisphere and infratentorial & 130 & 45 & 3 & 4 \\
\hline 10 & $40-44$ & Bilateral & 21 & 97 & 3 & 4 \\
\hline 11 & $75-79$ & Bilateral & 14 & 45 & 6 & 2 \\
\hline 12 & $15-19$ & Bilateral & 32 & 144 & NA & 4 \\
\hline 13 & $45-49$ & Bilateral & 19 & 139 & 3 & 1 \\
\hline 14 & $45-49$ & Bilateral & 19 & 101 & 7 & 1 \\
\hline 15 & $45-49$ & Bilateral and infratentorial & 12 & 23 & 12 & 1 \\
\hline 16 & $45-49$ & Bilateral & 30 & 23 & 7 & 4 \\
\hline 17 & $35-39$ & Bilateral and infratentorial & 20 & 147 & 3 & 4 \\
\hline 18 & $75-79$ & Bilateral and infratentorial & 16 & 31 & 8 & 1 \\
\hline
\end{tabular}

M, male; F, female; CT, computed tomography; EEG, electroencephalography; GCS, Glasgow Coma Scale; GOSE, Extended Glasgow Outcome Scale; NA, not available.

ranged from 0.02 to $0.08 \mathrm{~Hz}$. Phase-amplitude coupling was often seen to some extent, both in stroke and in trauma patients. In general, increased ISA periodicity was associated with increased phase-amplitude coupling (Figures 4 and 5). With increased ISA periodicity, we also observed an increase of pathological delta activity.

As expected, asymmetry was present in patients with hemispheric brain infarcts. In the conventional frequency band, this was mainly due to increased pathological delta power in the lesioned hemisphere. ISA amplitude, frequency and periodicity distributions appeared to be essentially the same in both hemispheres.

\section{No Successful CSD Detection}

Relevant changes of ISA were always visible in multiple electrodes, simultaneously. Often, ISA appeared as "sharp negative spikes" on all positions and was accompanied by an increase of delta power. This activity was always seen in both hemispheres, simultaneously (Figures 1 and 3). There were no focal SPC-like phenomena, or SPCs that were accompanied by suppression of faster brain activity, as expected in CSD.

In all patients, drops of high amplitude delta activity were observed (Figure 3). However, these depressions of spontaneous brain activity were not accompanied by simultaneous SPC-like phenomena. Moreover, these always occurred 

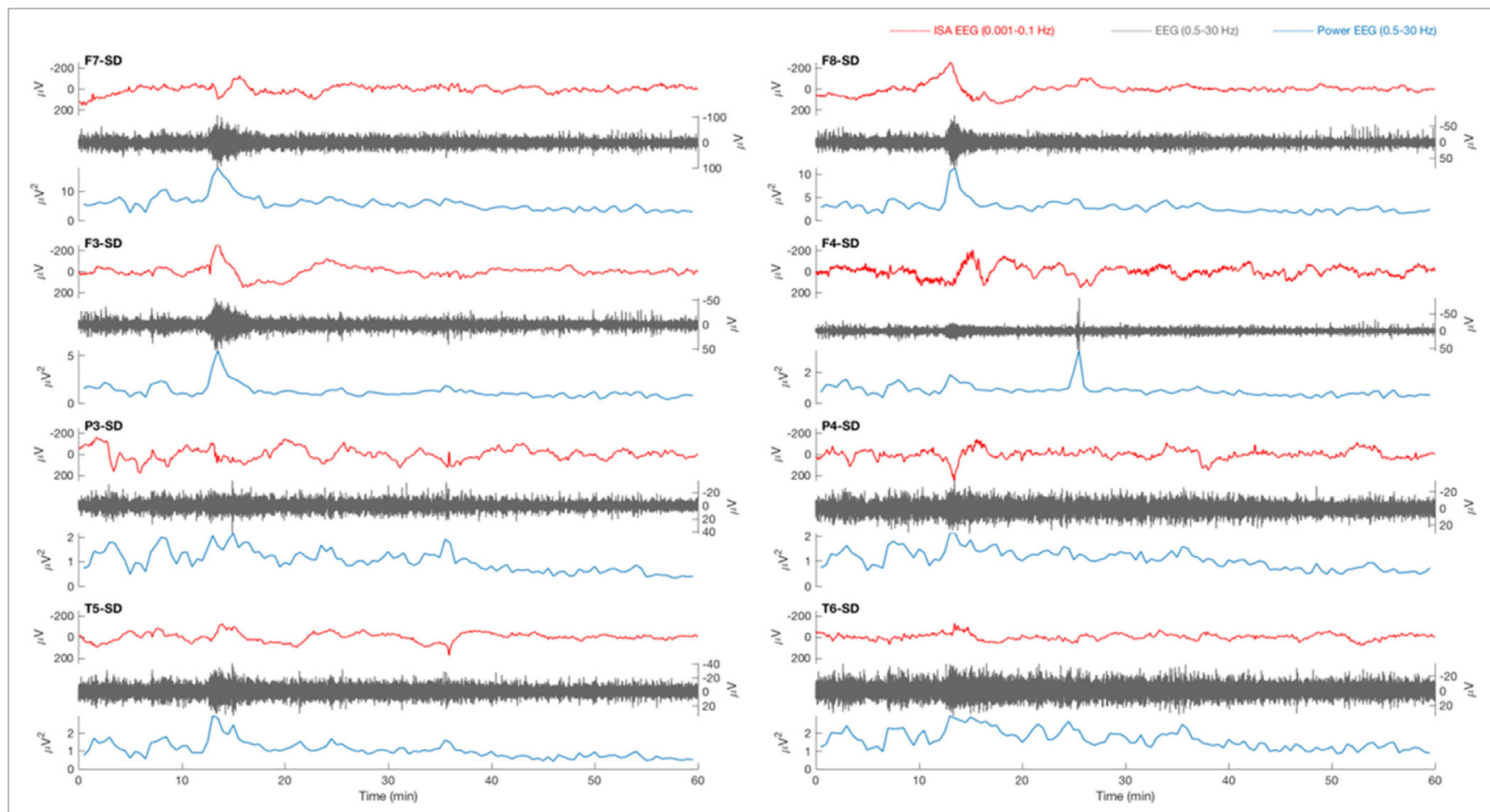

FIGURE 1 | Typical example of a $1 \mathrm{~h}$ electroencephalography (EEG) overview from patient 18 with traumatic brain injury. Source derivation for four electrodes (F7, F3, P3, and T5) for the left hemisphere and four electrodes (F8, F4, P4, and T6) for the right hemisphere are displayed. The infraslow activity (ISA, 0.001-0.1 Hz, red) shows a slow potential change (SPC) at minute 13 on positions F7, F3, F8, F4, and P4. On other positions, the activity is less prominent. A simultaneous increase of the amplitude (gray) and power (blue) of faster EEG activity $(0.5-30 \mathrm{~Hz}$, gray) can be seen in all electrodes. The SPC is not focal, not accompanied by a depression of spontaneous activity, and there is no delay between appearances on the various electrodes, as expected in cortical spreading depolarization (CSD).

simultaneously on many channels and were not focal, let alone spread, as expected in CSD. In sum, no CSD candidate was identified.

\section{DISCUSSION}

We were unable to identify unambiguous characteristics of CSD in patients with cortical ischemic stroke or traumatic brain injury, despite careful inspection of 3,035 h of continuous full band EEG in the infraslow and conventional frequency bands. This indicates that prospective CSD detection by full band scalp EEG is difficult. Of note, the inability to detect CSD does not undermine our belief in its existence and relevance.

Previous researchers have implicated identification of EEG signatures of CSD in patients with subarachnoid hemorrhage (24), malignant MCA infarction (24), or traumatic brain injury (27). Identification of CSD was always based on simultaneous recordings with subdural ECoG electrodes in patients that had been subject to craniotomy. After having identified depolarizations in ECoG recordings, scalp EEG correlates were examined by retrospective review of time aligned data. Unsupported by ECoG, in patients without craniotomy, we were unable to detect possible CSD with EEG. Factors that may be associated with this inability include artifacts, limited spatial resolution, inadequate visual analysis, and true inability to detect CSD with scalp EEG alone. These are discussed below.
By visual inspection of the complete, unfiltered EEG datasets, we observed various artifacts, including movement- and nursing procedure artifacts, contact phenomena, and changes of the redox potential at the electrode-skin interface. Artifacts influenced the infraslow components of the EEG more than the conventional frequency bands. Moreover, in lower frequency bands, EEG recovery from artifacts was slower. Especially in freely moving patients at the stroke unit, abundant artifacts in the ISA frequency bands hampered proper visual analysis. Artifacts in the ISA band are especially relevant for detection of isoelectric spreading depolarizations, i.e., spreading depolarizations in brain areas where conventional EEG activity is already depressed. In these cases, detection of spreading depolarization completely depends on detection of an SPC (24).

We used 21 conventional electrode positions with some additional electrodes above the assumed penumbra in patients with brain infarcts. The COSBID group EEG data on CSD were obtained with the same electrode configuration. Unlike in their ECoG data, in their EEG data, spread of amplitude depression was noticed in only a minority of patients with traumatic brain injury (27), and not in patients with brain infarcts (24). This may result from superposition of volume conducted EEG signals from widespread cortical generators (24). Otherwise, it may indicate that spatial resolution of a conventional EEG set up is insufficient to detect CSD, and higher density arrays are needed. 

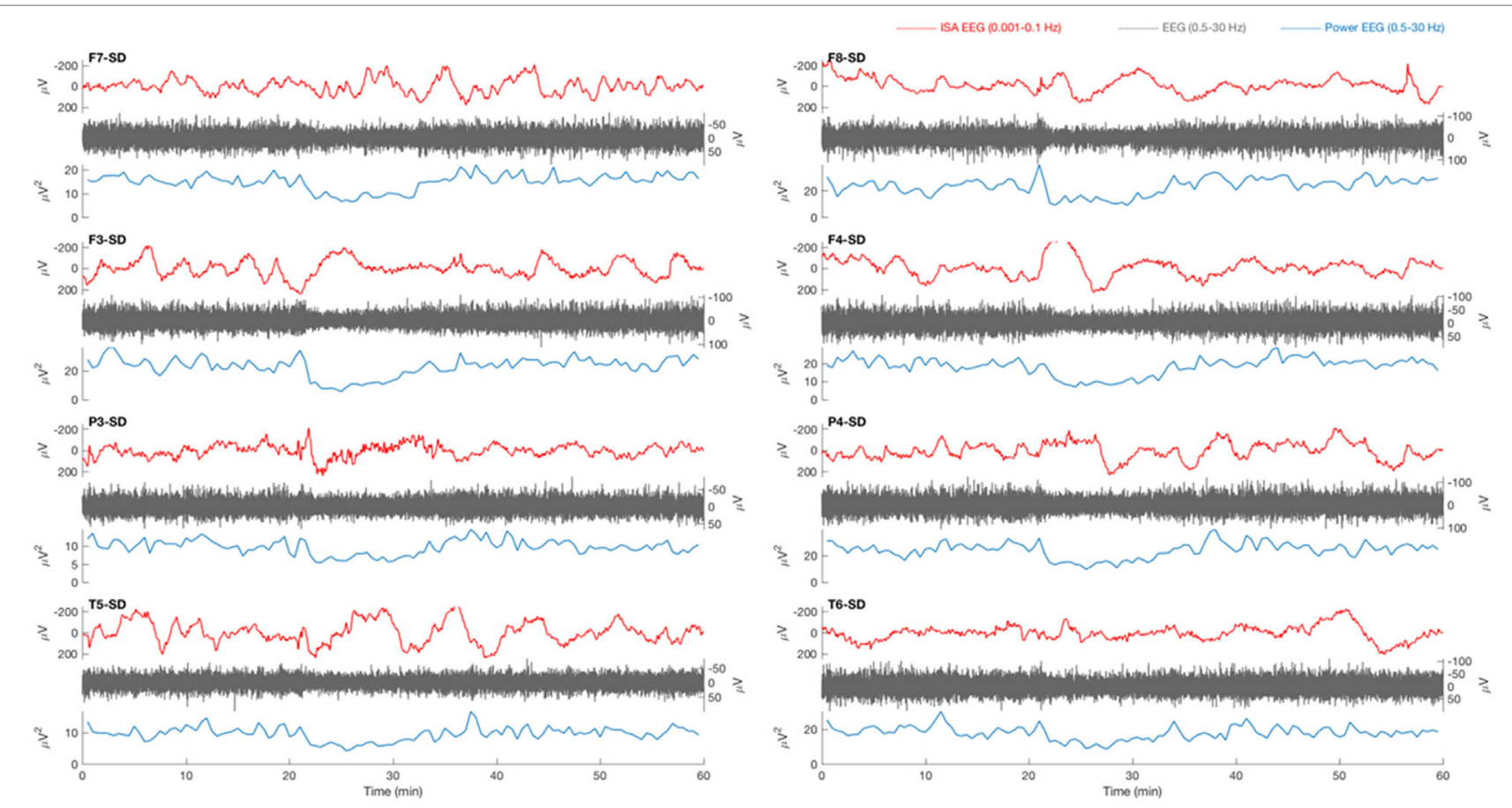

FIGURE 2 | Typical example of a 1 h electroencephalography (EEG) overview from patient 8 with traumatic brain injury. Source derivations for four electrodes (F7, F3, P3, and T5) of the left hemisphere and four electrodes (F8, F4, P4, and T6) of the right hemisphere are displayed. At 20 min, in the infraslow frequency (ISA) band $(0.001-0.1 \mathrm{~Hz}$, red), a slow potential change (SPC) is present on all electrode positions except F7 and T6. The SPC is accompanied by a depression of spontaneous activity $(0.5-30 \mathrm{~Hz}$, gray), which is clearly visible in the power of this frequency band (blue). The suppression is not focal, as expected in cortical spreading depolarization (CSD), but occurs on all positions simultaneously.

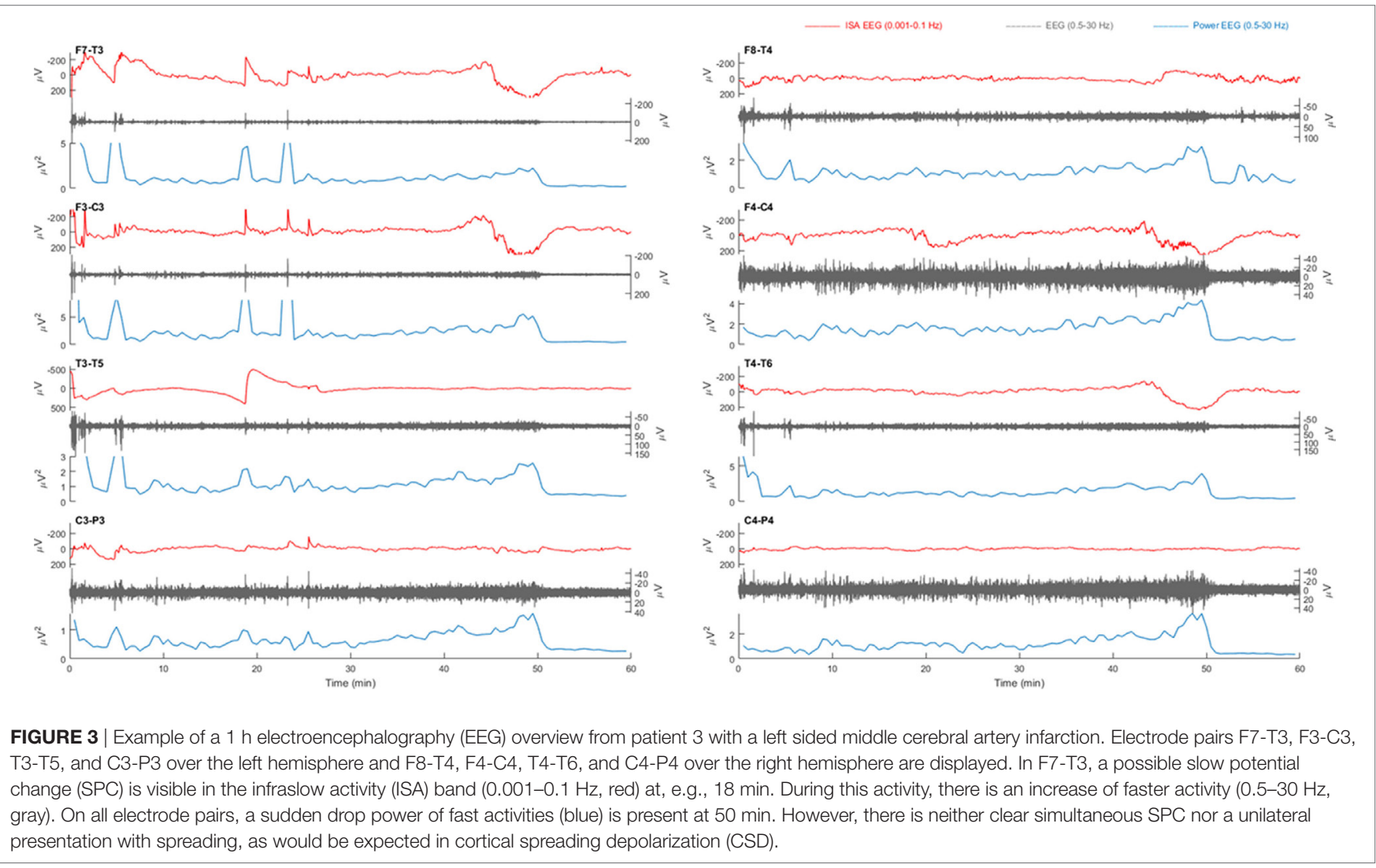



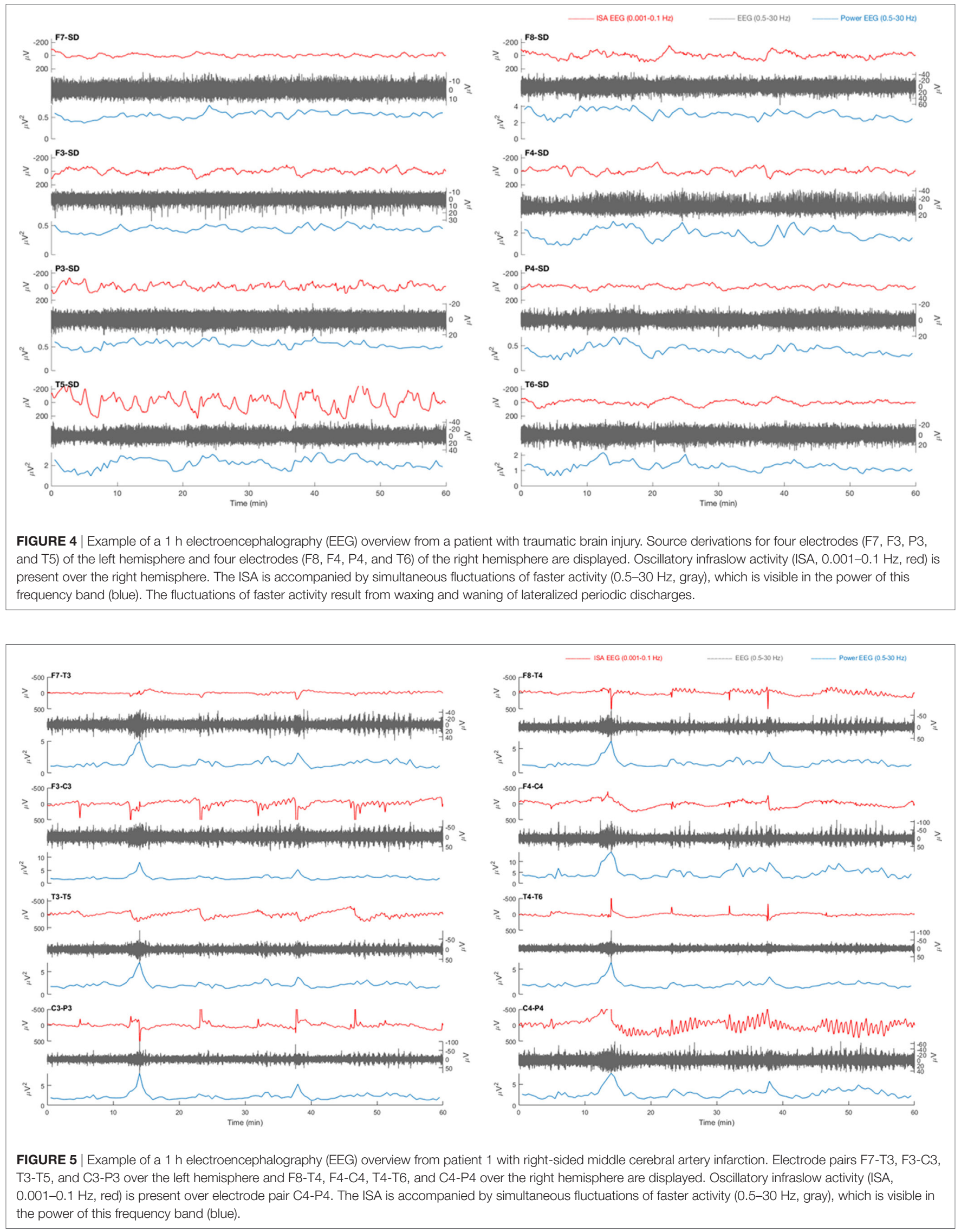
Even if we waive the criterion of spread, we were unable to identify candidate phenomena, since we did not observe the combination of clear SPC and simultaneous, focal amplitude depression of faster activity. Contrary, most features resembling SPC were accompanied by amplitude increases of faster activities. ISA associated with faster neuronal activities has been recognized since the late 1950s (28). There are roughly two views on the pathophysiology of this association. First, these could be an emergent property of the fast oscillations and thus be completely generated cortically. Second, these may result from distant mechanisms influencing cortical membrane potentials. Since CSD is a cortical phenomenon, CSD-associated SPCs should be generated cortically. This possibility is supported by the notion that negative potential shifts can arise as byproducts of fast activities (29). However, there is far more evidence supporting distant ISA generators, mostly subcortically, influencing cortical rhythms by modulation of cortical excitability (29). It is not possible to differentiate between the two mechanisms from ECoG or EEG measurements alone. Moreover, in higher mammals such as cats and monkeys, and hence also likely in humans, there may be a complex relationship between the scalp direct current potential and cerebral blood flow changes (30) or changes in partial pressures of $\mathrm{CO}_{2}$ or $\mathrm{O}_{2}(31,32)$ at the blood-brain barrier. Therefore, and since the criterion of spread was not fulfilled in any of our and most of the COSBID measurements, in the absence of a validated gold standard for CSD detection we doubt the true nature of all reported phenomena.

Our study obviously has limitations. First, we analyzed all EEG data by visual inspection. We chose to do so, because visual inspection still is considered gold standard for EEG interpretation. This even applies to artifact detection algorithms, which have been validated against visual analyses, once. We assumed that, for clinically relevant EEG-based CSD detection, it should be possible to visually identify at least some CSD signatures. Our methods and findings do not exclude subtle effects in the EEG, to be identified by advanced signal processing. However, the nature of such effects, if identified, would remain uncertain and preclude use at the bedside. We therefore believe that our findings call into question the possibility of clinically relevant CSD detection by scalp EEG. Second, interpretation of ISA, oscillatory or not, was hampered by lack of data from a healthy population. Third, in trauma patients, effects of sedative medication are not taken into account.

\section{REFERENCES}

1. Leao AA. Further observations on the spreading depression of activity in the cerebral cortex. J Neurophysiol (1947) 10:409-14. doi:10.1152/jn.1947.10.6.409

2. Zandt BJ, Ten Haken B, Van Putten MJAM, Dahlem MA. How does spreading depression spread? Physiology and modeling. Rev Neurosci (2015) 26:183-98. doi:10.1515/revneuro-2014-0069

3. Shinohara M, Dollinger B, Brown G, Rapoport S, Sokoloff L. Cerebral glucose utilization: local changes during and after recovery from spreading cortical depression. Science (1979) 203:188-90. doi:10.1126/science.758688

4. Somjen GG. Ions in the Brain: Normal Function, Seizures and Stroke. New York: Oxford University Press (2004).

5. Nedergaard M, Hansen AJ. Spreading depression is not associated with neuronal injury in the normal brain. Brain Res (1988) 449:395-8. doi:10.1016/ 0006-8993(88)91062-1

6. Lauritzen M. Pathophysiology of the migraine aura. The spreading depression theory. Brain (1994) 117(Pt 1):199-210. doi:10.1093/brain/117.1.199
In conclusion, with full band scalp EEG in patients with cortical ischemic stroke and traumatic brain injury, we observed various ISA, probably modulating cortical excitability. However, we were unable to identify unambiguous characteristics of CSD. We support the recently published consensus statement that invasive recordings are needed for detection of CSD and, so far, scalp EEG alone is not sufficient (33).

\section{ETHICS STATEMENT}

The Medical Research Ethics Committee Twente approved the research protocol for monitoring and follow-up of patients with ischemic stroke in October 2014 (registry number NL50284.044.14). Informed consent for continuous EEG monitoring and follow-up patients with ischemic stroke was obtained from the patient or a legal representative, in case of a decreased consciousness or severe aphasia. Since continuous EEG monitoring of patients with a decreased consciousness is part of current care on the intensive care unit of MST, the Medical Research Ethics Committee Twente waived the need for informed consent in patients with acute traumatic brain injury.

\section{AUTHOR CONTRIBUTIONS}

JH: study design and conceptualization, data interpretation, and writing first draft; CK and BW: data collection, data analysis, and interpretation and revising the manuscript for intellectual content; SV: data collection and revising the manuscript for intellectual content; MT-C and MP: study design and conceptualization and data interpretation. All the authors: final approval and agreement to be accountable.

\section{ACKNOWLEDGMENTS}

The authors thank all clinical neurophysiology lab technicians from Rijnstate Hospital and Medisch Spectrum Twente.

\section{FUNDING}

This research received no specific grant from any funding agency in the public, commercial, or not-for-profit sectors.

7. Lauritzen M, Dreier JP, Fabricius M, Hartings JA, Graf R, Strong AJ. Clinical relevance of cortical spreading depression in neurological disorders: migraine, malignant stroke, subarachnoid and intracranial hemorrhage, and traumatic brain injury. J Cereb Blood Flow Metab (2011) 31:17-35. doi:10.1038/ jcbfm.2010.191

8. Hadjikhani N, Sanchez Del Rio M, Wu O, Schwartz D, Bakker D, Fischl B, et al. Mechanisms of migraine aura revealed by functional MRI in human visual cortex. Proc Natl Acad Sci U S A (2001) 98:4687-92. doi:10.1073/pnas. 071582498

9. Olesen J, Larsen B, Lauritzen M. Focal hyperemia followed by spreading oligemia and impaired activation of rCBF in classic migraine. Ann Neurol (1981) 9:344-52. doi:10.1002/ana.410090406

10. Fabricius M, Fuhr S, Bhatia R, Boutelle M, Hashemi P, Strong AJ, et al. Cortical spreading depression and peri-infarct depolarization in acutely injured human cerebral cortex. Brain (2006) 129:778-90. doi:10.1093/brain/awh716

11. Strong AJ, Fabricius M, Boutelle MG, Hibbins SJ, Hopwood SE, Jones R, et al. Spreading and synchronous depressions of cortical activity in 
acutely injured human brain. Stroke (2002) 33:2738-43. doi:10.1161/01. STR.0000043073.69602.09

12. Ayata C, Shin HK, Salomone S, Ozdemir-Gursoy Y, Boas DA, Dunn AK, et al. Pronounced hypoperfusion during spreading depression in mouse cortex. J Cereb Blood Flow Metab (2004) 24:1172-82. doi:10.1097/01.WCB. 0000137057.92786.F3

13. Hinzman JM, Andaluz N, Shutter LA, Okonkwo DO, Pahl C, Strong AJ, et al. Inverse neurovascular coupling to cortical spreading depolarizations in severe brain trauma. Brain (2014) 137:2960-72. doi:10.1093/brain/awu241

14. Dohmen C, Sakowitz OW, Fabricius M, Bosche B, Reithmeier T, Ernestus R-I, et al. Spreading depolarizations occur in human ischemic stroke with high incidence. Ann Neurol (2008) 63:720-8. doi:10.1002/ana.21390

15. Hartings JA, Strong AJ, Fabricius M, Manning A, Bhatia R, Dreier JP, et al. Spreading depolarizations and late secondary insults after traumatic brain injury. J Neurotrauma (2009) 26:1857-66. doi:10.1089/neu.2009.0961

16. Hartings JA, Bullock MR, Okonkwo DO, Murray LS, Murray GD, Fabricius M, et al. Spreading depolarisations and outcome after traumatic brain injury: a prospective observational study. Lancet Neurol (2011) 10:1058-64. doi:10.1016/ S1474-4422(11)70243-5

17. Winkler MK, Dengler N, Hecht N, Hartings JA, Kang EJ, Major S, et al. Oxygen availability and spreading depolarizations provide complementary prognostic information in neuromonitoring of aneurysmal subarachnoid hemorrhage patients. J Cereb Blood Flow Metab (2017) 37:1841-56. doi:10.1177/0271678X16641424

18. Hartings JA, York J, Carroll CP, Hinzman JM, Mahoney E, Krueger B, et al. Subarachnoid blood acutely induces spreading depolarizations and early cortical infarction. Brain (2017) 140:2673-90. doi:10.1093/brain/ awx214

19. Dreier JP, Major S, Manning A, Woitzik J, Drenckhahn C, Steinbrink J, et al. Cortical spreading ischaemia is a novel process involved in ischaemic damage in patients with aneurysmal subarachnoid haemorrhage. Brain (2009) 132:1866-81. doi:10.1093/brain/awp102

20. Dreier JP. The role of spreading depression, spreading depolarization and spreading ischemia in neurological disease. Nat Med (2011) 17:439-47. doi: $10.1038 / \mathrm{nm} .2333$

21. Woitzik J, Hecht N, Pinczolits A, Sandow N, Major S, Winkler MKL, et al. Propagation of cortical spreading depolarization in the human cortex after malignant stroke. Neurology (2013) 80:1095-102. doi:10.1212/ WNL.0b013e3182886932

22. Dreier JP, Woitzik J, Fabricius M, Bhatia R, Major S, Drenckhahn C, et al. Delayed ischaemic neurological deficits after subarachnoid haemorrhage are associated with clusters of spreading depolarizations. Brain (2006) 129:3224-37. doi:10.1093/brain/awl297

23. Helbok R, Schiefecker AJ, Friberg C, Beer R, Kofler M, Rhomberg P, et al. Spreading depolarizations in patients with spontaneous intracerebral hemorrhage: association with perihematomal edema progression. J Cereb Blood Flow Metab (2017) 37:1871-82. doi:10.1177/0271678X16651269

24. Drenckhahn C, Winkler MKL, Major S, Scheel M, Kang E-J, Pinczolits A, et al. Correlates of spreading depolarization in human scalp electroencephalography. Brain (2012) 135:853-68. doi:10.1093/brain/aws010

25. Kemp B, van Beelen T, Stijl M, van Someren P, Roessen M, van Dijk JG. A DC attenuator allows common EEG equipment to record fullband EEG, and fits fullband EEG into standard European Data Format. Clin Neurophysiol (2010) 121:1992-7. doi:10.1016/j.clinph.2010.05.006

26. Welch PD. The use of fast Fourier transform for the estimation of power spectra: a method based on time averaging over short, modified periodograms. IEEE (1967) 15:70-3. doi:10.1109/TAU.1967.1161901

27. Hartings JA, Wilson JA, Hinzman JM, Pollandt S, Dreier JP, DiNapoli V, et al. Spreading depression in continuous electroencephalography of brain trauma. Ann Neurol (2014) 76:681-94. doi:10.1002/ana.24256

28. Aladjalova NA. Infra-slow rhythmic oscillations of the steady potential of the cerebral cortex. Nature (1957) 179:957-9. doi:10.1038/179957a0

29. Palva JM, Palva S. Infra-slow fluctuations in electrophysiological recordings, blood-oxygenation-level-dependent signals, and psychophysical time series. Neuroimage (2012) 62:2201-11. doi:10.1016/j.neuroimage.2012.02.060

30. Besson JM, Woody CD, Aleonard P, Thompson HK, Albe-Fessard D, Marshall WH. Correlations of brain d-c shifts with changes in cerebral blood flow. Am J Physiol (1970) 218:284-91. doi:10.1152/ajplegacy.1970.218.1.284

31. Lehmenkühler A, Richter F, Pöppelmann T. Hypoxia- and hypercapniainduced DC potential shifts in rat at the scalp and the skull are opposite in polarity to those at the cerebral cortex. Neurosci Lett (1999) 270:67-70. doi:10.1016/S0304-3940(99)00469-3

32. Voipio J, Tallgren P, Heinonen E, Vanhatalo S, Kaila K. Millivolt-scale DC shifts in the human scalp EEG: evidence for a nonneuronal generator. J Neurophysiol (2003) 89:2208-14. doi:10.1152/jn.00915.2002

33. Dreier JP, Fabricius M, Ayata C, Sakowitz OW, William Shuttleworth C, Dohmen C, et al. Recording, analysis, and interpretation of spreading depolarizations in neurointensive care: review and recommendations of the COSBID research group. J Cereb Blood Flow Metab (2017) 37:1595-625. doi:10.1177/ $0271678 X 16654496$

Conflict of Interest Statement: MP is cofounder of Clinical Science Systems. The other authors report to have no conflicts of interest.

Copyright $\odot 2018$ Hofmeijer, van Kaam, van de Werff, Vermeer, Tjepkema-Cloostermans and van Putten. This is an open-access article distributed under the terms of the Creative Commons Attribution License (CC BY). The use, distribution or reproduction in other forums is permitted, provided the original author(s) or licensor are credited and that the original publication in this journal is cited, in accordance with accepted academic practice. No use, distribution or reproduction is permitted which does not comply with these terms. 